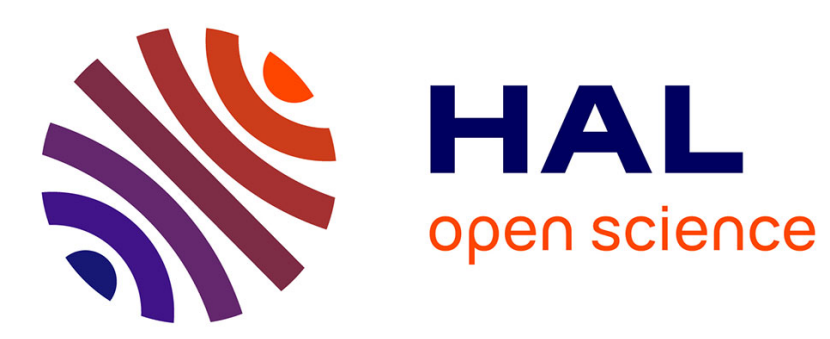

\title{
Intérêt des aliments fermentés pour la santé
}

Isabelle Savary-Auzeloux, Françoise Rul

\section{To cite this version:}

Isabelle Savary-Auzeloux, Françoise Rul. Intérêt des aliments fermentés pour la santé. Pratiques en Nutrition: santé et alimentation, 2021, 17 (68), pp.8-12. 10.1016/j.pranut.2021.09.003 . hal03455468

\section{HAL Id: hal-03455468 \\ https://hal.science/hal-03455468}

Submitted on 29 Nov 2021

HAL is a multi-disciplinary open access archive for the deposit and dissemination of scientific research documents, whether they are published or not. The documents may come from teaching and research institutions in France or abroad, or from public or private research centers.
L'archive ouverte pluridisciplinaire HAL, est destinée au dépôt et à la diffusion de documents scientifiques de niveau recherche, publiés ou non, émanant des établissements d'enseignement et de recherche français ou étrangers, des laboratoires publics ou privés. 


\title{
L'intérêt des aliments fermentés pour la santé : quelles bases ?
}

\author{
Isabelle Savary-Auzeloux ${ }^{\mathrm{a}, *}$
}

Directrice de recherches INRAE

\section{Françoise Rul ${ }^{\mathbf{b}}$}

Chargée de recherches INRAE

aUniversité Clermont Auvergne, INRAE - UMR 1019 Unité de Nutrition Humaine, 63000 Clermont-Ferrand, France

${ }^{\mathrm{b}}$ UMR Micalis-AgroParisTech, Université Paris-Saclay, 78350 Jouy-en-Josas

\section{*Auteur correspondant.}

Adresse e-mail : isabelle.savary-auzeloux@inrae.fr (I. Savary-Auzeloux).

\section{Résumé}

La fermentation permet de conserver de manière durable les aliments à moindre coût dans de nombreux pays et participe grandement à la sécurité alimentaire mondiale. Ce mode de conservation est actuellement très prisé par une population en recherche de naturalité. Des vertus santé sont également attribuées aux aliments fermentés parce qu'ils contiennent des microorganismes vivants ou des macro-micronutriments spécifiques. Ces produits, très divers, ou le processus de fermentation en tant que tel sont-ils bénéfiques pour la santé ?

\section{Mots clés -}

La fermentation est, avec le salage et le séchage, la méthode la plus ancienne de conservation des aliments puisqu'elle remonte à un peu moins de 10000 ans avant notre ère $(1,2)$. C'est un procédé simple, durable, qui ne fait pas intervenir la chaîne du froid et qui est réalisable à petite et à grande échelle, ce qui explique que les aliments fermentés peuvent être fabriqués à la fois par l'industrie agroalimentaire et de manière artisanale.

À partir de matières premières extrêmement variées, des communautés microbiennes endogènes et/ou ajoutées vont se développer et générer un produit très éloigné de la matière première de départ. Le procédé technologique de fermentation, qui est largement répandu dans le monde, permet d'élaborer des aliments très différents selon les conditions environnementales (température, humidité, etc.), les micro-organismes présents dans l'environnement ou ajoutés, et les matières premières à disposition sur le site de production. À titre d'exemple, si les Européens sont très friands de yaourts, de fromages ou autres produits laitiers fermentés, les Asiatiques apprécient de nombreux produits fermentés à base de végétaux, comme le tofu, le kombucha ou le kimchi. Puisque ces aliments couvrent une gamme extrêmement large, ils diffèrent également fortement en termes de texture (de la sauce soja au fromage, en passant par la choucroute), de goût (produits doux, acides, amers, etc.) et de composition en flore (bactéries, levures et champignons). 
Depuis quelques années, de nombreux blogs et ouvrages se sont approprié ce mode de conservation millénaire pour en faire un sujet tendance, très prisé par une population soucieuse de durabilité, de maîtrise des procédés et de produits plus naturels, moins industriels, sans additifs et faits maison (https://www.vitagora.com/en/blog/2019/fermented-products-french-market/). Ce marché est porteur (plus de 600 milliards de dollars en 2016-2018) et en expansion de plus de $5 \%$ par an (BIS Research). II est par conséquent très scruté par l'industrie agroalimentaire et les start-ups, à la recherche d'idées nouvelles pour la grande distribution ou les marchés de niche. Or, malgré cet engouement indéniable, une étude Nutrimarketing en 2018 a montré que seuls $44 \%$ des sondés connaissaient le procédé de fermentation et que $60 \%$ avaient une perception négative du terme "fermenté", alors qu'ils mangeaient tous du fromage. La fermentation et les aliments qui en sont issus sont donc largement méconnus de la population générale.

En parallèle de ces considérations éthiques, culturelles et économiques, il apparaît qu'il est attribué aux aliments fermentés des actions bénéfiques sur la santé intestinale et la santé en général. Quand on connaît la difficulté d'objectiver l'effet santé d'un aliment seul ou de diètes (études cliniques ou observationnelles nombreuses, parfois contradictoires), l'attribution d'un tel effet aux produits fermentés dans leur ensemble peut donc sembler illusoire. Néanmoins, il reste intéressant de s'interroger sur les vertus santé qui leur sont prêtées. Ne concernent-elles que certains aliments ou le processus de fermentation ? Si cet effet est avéré, sur quelles cibles ces produits agissent-ils ?

\section{Définitions et caractéristiques des aliments fermentés}

Pour répondre à ces questions complexes, il convient dans un premier temps d'expliciter ce que l'on entend par processus de fermentation d'une grande gamme de matières premières et de faire un état des lieux de toute la diversité des produits fermentés générés.

\section{Définitions}

Une revue de l'Association scientifique internationale des probiotiques et des prébiotiques (2) a très récemment fait le point sur la définition qui peut être attribuée aux aliments fermentés ainsi qu'à leur rôle dans l'alimentation humaine. D'un point de vue biochimique, la fermentation se définit comme "un processus générant de I'ATP [adénosine triphosphate] dans lequel les composés organiques agissent à la fois en tant que donneurs et accepteurs d'électrons ". Cette définition s'applique bien aux fermentations anaérobies lactiques ou alcooliques (yaourt, vin, etc.), mais pas aux produits faisant appel à des fermentations aérobies, telles que celles mises en œuvre dans la production de la sauce soja (champignons) ou le vinaigre (bactéries acétiques). Par conséquent, les experts ont retenu une définition plus large pour décrire les aliments et les boissons fermentés: "Aliments obtenus suite à la croissance désirée de microbes et de conversions enzymatiques de matières premières alimentaires » (2). Ainsi, la présence $d^{\prime}$ enzymes endogènes ou exogènes 
des matières premières ne peut, à elle seule, être suffisante pour qu'un aliment soit dit fermenté.

À titre d'exemple, on peut ainsi considérer comme fermentés les produits dans lesquels les microorganismes utilisés pour la fermentation sont présents vivants (la plupart des fromages et des yaourts, le miso, certaines bières, le kéfir, etc.) ou morts (pain au levain, produits fermentés puis pasteurisés, la plupart des vins et des spiritueux, etc.). En revanche, ce n'est pas le cas de tous ceux issus de la salaison seule, les végétaux en saumure et le pain obtenu avec de la levure chimique.

\section{Une grande diversité}

Plus de 5000 aliments fermentés sont référencés (tableau 1) (1, 3-6). Les matières premières animales (lait, viandes, poissons) et végétales (céréales, légumineuses, tubercules, racines, fruits, légumes, graines et fèves) sont susceptibles de subir un processus de fermentation pour donner toute une gamme de produits.

Les microorganismes qui interviennent dans ce procédé peuvent avoir des origines variées : ils sont ajoutés extemporanément (amorçage, beaucoup utilisé dans les industries agroalimentaires car l'ensemencement peut être strictement contrôlé), repiqués à partir de produits fermentés précédemment (repiquage) ou sont naturellement présents dans la matière première (croissance spontanée ${ }^{1}$ ). Ce sont essentiellement des bactéries lactiques et acétiques, mais d'autres types bactériens sont parfois présents - les levures et les champignons filamenteux -, surtout lorsqu'il s'agit d'aliments à ensemencement spontané.

Puisque l'aliment fermenté est le résultat de l'association d'une matière première et de microorganismes variés, le nombre de combinaisons est quasiment infini, ce qui explique la très grande diversité de ces produits.

\section{Des particularités uniques}

En dehors des considérations microbiologiques, ces produits présentent également un profil biochimique unique lié à la fermentation. En effet, ce procédé se traduit par la synthèse de molécules à partir de celles présentes dans la matière première : peptides antihypertenseurs, acides gras à chaîne courte, vitamines du groupe $B$, acides gras polyinsaturés de type oméga-3 notamment $(7,8)$. II engendre également la dégradation de composés présents dans la matière première: lactose, protéines allergènes, acide phytique, Fermentable oligo, di-, mono-mers and polyols $(7,8)$, etc.

Les caractéristiques physico-chimiques des aliments fermentés sont souvent très différentes de celles de la matière première initiale : $\mathrm{pH}$, acidité, potentiel redox ou activité antioxydante, micro-macrostructure. Par conséquent, la texture, le goût, la sensation en bouche de ces denrées sont très éloignés de ceux de la matière première dont ils sont issus.

\section{Fermentation, sécurité alimentaire et risques potentiels}


La fermentation, procédé unique utilisé depuis des millénaires, a pour objectif principal de maintenir comestibles des matières premières sur une durée plus longue. Néanmoins, ce processus de conservation peut présenter certains risques qu'il convient de ne pas négliger.

\section{Implication dans la sécurité alimentaire}

Puisque la fermentation permet la préservation des matières premières, il est évident que les aliments fermentés jouent un rôle majeur en santé publique, en particulier dans les pays chauds et dans ceux où les autres modes de préservation (comme le froid) sont peu répandus. Ces produits contribuent donc parfois à couvrir les besoins nutritionnels de base des populations.

Un aliment fermenté peut être considéré sûr s'il contient des concentrations en acides organiques supérieur à $100 \mathrm{mM}$ combinées avec une faible quantité d'eau, du sel, des nitrites et d'autres composés antimicrobiens (2). C'est aussi le cas des boissons qui renferment plus de $4 \%$ d'alcool et dont le $\mathrm{pH}$ est inférieur à 4,5. En outre, les bactéries lactiques présentent la particularité de synthétiser des bactériocines inhibant la croissance d'autres bactéries.

La fermentation permet de dégrader certains composés qui peuvent avoir des effets néfastes sur la santé. Un des avantages les plus connus de ce procédé est la dégradation du lactose, naturellement présent dans le lait, par les bêta-galactosidases bactériennes (9). D'ailleurs, la seule allégation santé actuellement autorisée par l'European Food Safety Authority concernant les aliments fermentés est l'ingestion du yaourt pour les populations intolérantes au lactose (EFSA, 2010). La dégradation, par la fermentation, des composés indésirables ou antinutritionnels, comme l'acide phytique, les alcaloïdes, les inhibiteurs trypsiques et les composés cyanogènes, est également connue (10); elle peut limiter la toxicité directe de certaines de ces molécules (glycosides cyanogènes), favoriser l'absorption des autres nutriments alimentaires (absorption du calcium et du magnésium pour l'acide phytique) ou minorer le caractère allergène de certaines protéines (11).

La fermentation permet aussi de valoriser certaines matières premières qui resteraient, sinon, sous-utilisées. C'est le cas notamment du café et du chocolat, mais aussi des végétaux chez lesquels la concentration en composés antinutritionnels est trop élevée pour que la digestion de l'ensemble des constituants de la diète se fasse correctement ou que la santé de l'hôte soit dégradée (acide phytique ou composés cyanogènes dans certaines céréales et légumineuses).

\section{Risques potentiels}

Les risques associés à l'ingestion des aliments fermentés sont de deux ordres : une possible contamination par des bactéries non désirées pathogènes et la synthèse, via la fermentation, de molécules connues pour être néfastes pour la santé. Les risques microbiologiques existent pour tous les produits fermentés, mais sont accrus lorsque le processus n'est pas complètement maîtrisé, mettant en jeu de la fermentation spontanée, généralement lorsqu'il est artisanal (12). Parmi les microorganismes pathogènes les plus 
connus, on peut citer Escherichia coli, Bacillus cereus, Listeria monocytogenes, Salmonella sp. et Clostridium botulinum. La possibilité que certains microorganismes présents dans les aliments fermentés puissent transmettre des gènes d'antibiorésistance fait l'objet d'un intérêt croissant.

Certaines molécules posent potentiellement un problème pour la santé, telles que, bien évidemment, l'alcool et le sel (ajouté dans certains aliments fermentés). Dans les deux cas, les conséquences se manifestent sur le long terme et se traduisent par des pathologies ou des dérèglements métaboliques majeurs (obésité, diabète, maladies cardio-vasculaires, stéatose hépatique). D'autres molécules peuvent avoir des effets plus aigus, comme les amines biogènes produites par les bactéries lactiques (migraines) (13) et les mycotoxines (aflatoxines, etc.) produites par les champignons filamenteux (intoxications aiguës avec lésions hépatiques par exemple) (https://www.anses.fr/fr/system/files/RCCP-RaMycotoxines2009.pdf)

\section{Objectivisation de l'effet santé chez l'homme}

La fermentation permet parfois de limiter l'apparition de molécules néfastes pour la santé et, à l'inverse, de favoriser celle de molécules potentiellement bénéfiques. Dans certains cas spécifiques (telle la dégradation du lactose), elle améliore la santé de certaines populations cibles. De nombreuses revues détaillent tous les impacts connus des molécules issues de la fermentation et les mécanismes d'interaction potentiels avec l'hôte pouvant engendrer des effets bénéfiques sur la santé au sens large $(14,15)$. Ces résultats ont généralement été obtenus sur des modèles animaux, plus rarement chez l'homme. Il en va de même pour l'étude du rôle des bactéries présentes dans ces produits et présentant des propriétés probiotiques $(16,17)$.

Via la connaissance de la composition en microorganismes et celle des métabolites et composés bénéfiques ou néfastes de ces aliments, il devrait être possible d'évaluer leur effet potentiel, positif ou négatif, sur la santé du consommateur. Mais il faudrait pouvoir prendre en compte les quantités ingérées, l'association de ces aliments avec les autres composants du repas, ainsi que les habitudes nutritionnelles et l'état de santé des populations considérées. Des études interventionnelles et observationnelles ont donc été réalisées chez l'homme, afin d'estimer, dans des conditions habituelles de vie, si l'ingestion de ces produits (ensemble ou individuellement) était capable d'influer sur la santé de l'hôte.

Peu d'effets avérés sur les pathologies chroniques et la santé intestinale

L'effet santé des aliments fermentés a été étudié, chez l'homme, dans les situations pathologiques ou les perturbations métaboliques suivantes: obésité, syndrome métabolique, diabète, maladies cardio-vasculaires, hypertension $(7,18)$. Leur rôle en cas de dérèglements ou de pathologies intestinales (allergies, pathologies inflammatoires, intolérances alimentaires, transit) est également documenté $(11,19)$. Une littérature moins abondante s'est aussi intéressée à la santé cognitive (20) et aux cancers (18). 
Concernant les effets globaux, il est bien connu que la diète méditerranéenne est associée à une amélioration de l'insulino-sensibilité et à une moindre occurrence de pathologies cardiovasculaires (21). Or, ce régime est connu pour être particulièrement riche en aliments fermentés, en particulier en produits laitiers fermentés qui contribueraient à son impact bénéfique (22).

Les produits laitiers peuvent être considérés à part, car leurs effets santé ont été largement étudiés dans de nombreux pays du monde. Ils ont fait l'objet de nombreuses méta-analyses et revues systématiques de la littérature, qui sont les analyses de données les plus robustes. Sur toutes les pathologies métaboliques considérées, seule la consommation de yaourt - et dans une moindre mesure celle de fromage - est négativement associée à l'apparition d'un diabète de type 2 (18), alors que l'ingestion du lait seul n'est pas corrélée positivement ou négativement avec cette pathologie. Cela suggère donc que la fermentation du lait per se est responsable du résultat observé sans que l'on sache avec certitude si son rôle bénéfique provient des bactéries présentes dans l'aliment, de sa composition en lipides ou d'effets liés à la matrice alimentaire qui sont tous très différents du lait natif. Pour les autres pathologies, les études manquent et/ou sont contradictoires pour valider de manière robuste un effet santé des produits laitiers fermentés.

Force est de constater que les données manquent pour objectiver le bénéfice sur la santé des autres produits fermentés, notamment végétaux. À titre d'exemple, il est conféré au soja dans son ensemble, ou aux protéines et aux isoflavones qu'il contient, des effets bénéfiques dans de nombreuses pathologies (maladies cardio-vasculaires, cancers, etc.) (23). Néanmoins, les produits à base de soja peuvent être fermentés (miso par exemple) ou non (jus de soja ou tofu), et rien ne prouve actuellement l'existence d'un impact positif spécifique à la fermentation les concernant. De manière générale, et pour de nombreux végétaux fermentés testés, les effets santés observés sont plutôt attribués aux macro(protéines, fibres) et micronutriments, naturellement présents dans la matière première ou ajoutés lors de la préparation de l'aliment (ajouts de composés antioxydants, tel l'ail pour la préparation du kimchi). À l'inverse, la version non fermentée de certains produits, comme le chocolat et le café, n'existe pas. Leurs effets santé peuvent donc être partiellement liés au processus de fermentation; là encore, la composition de la matière première (fibres, polyphénols, vitamines) semble être un des éléments explicatifs majeurs.

Néanmoins, lorsqu'on s'intéresse, sur des espèces modèles, à l'impact de molécules spécifiques (acides gras à chaîne courte, métabolites de dégradation des polyphénols, etc.) issues de la fermentation ou à l'effet de microorganismes particuliers (probiotiques), un impact bénéfique est retrouvé de manière plus systématique sur les pathologies nutriinduites, la santé intestinale et les cancers $(5,7)$. De ce fait, il ne peut être exclu que l'absence globale - sauf cas particuliers - d'effet santé avéré chez l'homme des produits fermentés peut être liée non pas à son absence, mais à notre difficulté à le mesurer chez l'homme par des marqueurs pertinents ou des schémas expérimentaux adaptés.

\section{Conclusion}


Les aliments fermentés font partie intégrante de notre diète depuis des millénaires. Ils présentent de nombreux avantages en termes de sécurité nutritionnelle en permettant la conservation, sur le long cours, de matières premières périssables de manière durable et très peu énergivore. Ce mode de conservation des aliments est aussi très prometteur en termes d'innovation, en substitution à d'autres méthodes de conservation des aliments moins durables. Néanmoins, du fait de la grande diversité des aliments fermentés, leur effet bénéfique sur la santé demeure peu étayé ${ }^{2}$. Ainsi, il reste nécessaire de mettre en place des études cliniques observationnelles ou interventionnelles pour lesquelles l'ingestion des aliments fermentés est parfaitement connue ou maîtrisée, combinées, d'une part, à des outils haut débit de phénotypage de la santé des individus et, d'autre part, à une connaissance approfondie des facteurs confondants (reste de la diète, quantités ingérées, état de santé des sujets, etc.). Seul ce type d'approche permettra d'objectiver avec certitude leurs impacts réels sur notre santé et déboucher, à plus long terme, sur des recommandations nutritionnelles spécifiques. 


\begin{tabular}{|c|c|c|c|c|c|}
\hline Type de produit & Matières premières & Nom du produit & $\begin{array}{l}\text { Microorganismes } \\
\text { impliqués }\end{array}$ & $\begin{array}{l}\text { Source des } \\
\text { microorganismes }\end{array}$ & Situation géographique \\
\hline \multirow[t]{2}{*}{ Produits laitiers } & $\begin{array}{l}\text { Lait } \\
\text { Lait, sel }\end{array}$ & $\begin{array}{l}\text { Yaourt } \\
\text { Fromage }\end{array}$ & $\begin{array}{l}B L \\
A B, B A c, C F, B L \\
\text { Lev selon les } \\
\text { fromages }\end{array}$ & $\begin{array}{l}\text { Amorçage } \\
\text { Amorçage, } \\
\text { repiquage }\end{array}$ & $\begin{array}{l}\text { Europe, Amérique } \\
\text { Europe, Amérique, } \\
\text { Océanie }\end{array}$ \\
\hline & Lait & $\begin{array}{l}\text { Lait fermenté (kéfir, lait } \\
\text { ribot, lassi, etc.) }\end{array}$ & Lev, BL, AB, B Ac & Repiquage & $\begin{array}{l}\text { Mondiale (sous } \\
\text { différents noms) }\end{array}$ \\
\hline \multirow[t]{4}{*}{ Fruits } & Fève de cacao & Chocolat & Lev, BL, AB, B Ac & Spontané & $\begin{array}{l}\text { Afrique, Amérique } \\
\text { latine, exportation } \\
\text { mondiale }\end{array}$ \\
\hline & Cerise de café & Café & Lev, CF, AB & Spontané & $\begin{array}{l}\text { Afrique, Amérique } \\
\text { latine, Asie, exportation } \\
\text { mondiale }\end{array}$ \\
\hline & Grains de raisin pressés & Vin & $\begin{array}{l}\text { Lev } \\
\text { essentiellement }\end{array}$ & $\begin{array}{l}\text { Spontané, } \\
\text { amorçage }\end{array}$ & Mondial \\
\hline & $\begin{array}{l}\text { Jus de pomme ou d'autres fruits, } \\
\text { eau (sucre pour kéfir) }\end{array}$ & Cidre, kéfir de fruits & $B L, A B$, Lev & $\begin{array}{l}\text { Amorçage, } \\
\text { repiquage }\end{array}$ & Mondial \\
\hline \multirow[t]{5}{*}{$\begin{array}{l}\text { Graines, racines, } \\
\text { tubercules }\end{array}$} & Orge, houblon, eau & Bière & $\begin{array}{l}\text { Lev } \\
\text { essentiellement }\end{array}$ & $\begin{array}{l}\text { Amorçage, } \\
\text { repiquage }\end{array}$ & Mondial \\
\hline & $\begin{array}{l}\text { Divers (riz, vin, pommes, pommes } \\
\text { de terre, orge, maïs, seigle, blé, } \\
\text { orge, etc.) }\end{array}$ & $\begin{array}{l}\text { Alcools forts : sake, cognac, } \\
\text { calvados, vodka, single malt } \\
\text { Whisky, bourbon, etc. }\end{array}$ & $\begin{array}{l}\text { Lev } \\
\text { essentiellement }\end{array}$ & $\begin{array}{l}\text { Spontané, } \\
\text { amorçage }\end{array}$ & Mondial \\
\hline & Farine de blé, eau & Pain au levain & Lev, BL & $\begin{array}{l}\text { Repiquage, } \\
\text { spontané }\end{array}$ & $\begin{array}{l}\text { Amérique, Europe, } \\
\text { Australie }\end{array}$ \\
\hline & Graine de soja, eau, sel & Sauce soja, miso & $\mathrm{CF}$ & $\begin{array}{l}\text { Amorçage, } \\
\text { spontané }\end{array}$ & $\begin{array}{l}\text { Asie, exportation } \\
\text { mondiale }\end{array}$ \\
\hline & $\begin{array}{l}\text { Graines de céréales, pseudo- } \\
\text { céréales, légumineuses }\end{array}$ & $\begin{array}{l}\text { Jus fermentés, tempe, } \\
\text { natto, idli, ben-saalga, ogi }\end{array}$ & CF, Lev, BL & Amorçage, & Asie, Afrique, \\
\hline
\end{tabular}




\begin{tabular}{|c|c|c|c|c|c|}
\hline & & & & spontané & exportation mondiale \\
\hline \multirow[t]{3}{*}{ Autres végétaux } & Chou, condiments, ail, sel & Kimchi & Lev, BL & $\begin{array}{l}\text { Spontané, } \\
\text { amorçage }\end{array}$ & $\begin{array}{l}\text { Asie, exportation } \\
\text { mondiale }\end{array}$ \\
\hline & Chou, sel & Choucroute & $B L$ & $\begin{array}{l}\text { Spontané, } \\
\text { amorçage }\end{array}$ & $\begin{array}{l}\text { Europe, Amérique, } \\
\text { Chine }\end{array}$ \\
\hline & Thé, sucre & Kombucha & Lev, BL, B Ac, AB & Repiquage & Asie, reste du monde \\
\hline Viandes & Viande, sel & Saucisson, salamis, jambons & Lev, CF, BL, AB, & $\begin{array}{l}\text { Amorçage, } \\
\text { spontané }\end{array}$ & $\begin{array}{l}\text { Europe, Amérique du } \\
\text { Nord, Asie }\end{array}$ \\
\hline Poissons, coquillages & $\begin{array}{l}\text { Poisson, sel, condiments } \\
\text { (fréquent), voire sucre }\end{array}$ & $\begin{array}{l}\text { Sauce nuoc-nâm, poissons } \\
\text { fermentés }\end{array}$ & Lev, BL, AB & Spontané & $\begin{array}{l}\text { Inde, Asie, Europe du } \\
\text { Nord, Grèce }\end{array}$ \\
\hline
\end{tabular}

Extrait et adapté de Wolfe et Dutton, $2015^{(4)}$, Marco et coll 2017 (5), Marshall et Meijia, 2011(1), Tamang 2020(6)

AB : autres bactéries ; B Ac : Bactéries acétiques ; BL : bactéries lactiques ; CF : champignons filamenteux ; Lev : levures.

Amorçage : ajout de microorganismes pour démarrer le processus de fermentation ; repiquage : utilisation des microorganismes issus de fermentations précédentes de la même matière première ; spontané : fermentation à partir des microorganismes présents dans la matière première

Tableau 1. Principales classes de produits fermentés, matières premières utilisées, microorganismes réalisant la fermentation, ainsi que lieu majeur de production et de consommation. 


\section{Note}

${ }^{1}$ Dans ce cas, la composition du consortium bactérien impliqué dans la fermentation n'est pas contrôlée, il s'agit donc souvent de procédés utilisés artisanalement ou chez soi. ${ }^{2}$ Produits laitiers mis à part ou situations dans laquelle la fermentation permet l'élimination ou l'apport de composés spécifiques, comme la dégradation du lactose ou des facteurs antinutritionnels.

\section{Références}

1. Marshall E, Meija D. Traditional fermented food and beverages for improved livehoods. FAO diversification booklet 21. 2011:90.

2. Marco ML, Sanders ME, Gänzle M, Arrieta MC, Cotter PD, De Vuyst L, et al. The International Scientific Association for Probiotics and Prebiotics (ISAPP) consensus statement on fermented foods. Nature reviews Gastroenterology \& hepatology. 2021.

3. Tamang JP, Kailasapathy K. Fermented Foods and Beverages of the World. CRC Press. 2010.

4. Wolfe BE, Dutton RJ. Fermented foods as experimentally tractable microbial ecosystems. Cell. 2015;161(1):49-55.

5. Marco ML, Heeney D, Binda S, Cifelli CJ, Cotter PD, Foligne B, et al. Health benefits of fermented foods: microbiota and beyond. Current opinion in biotechnology. 2016;44:94-102.

6. Tamang JP, Cotter PD, Endo A, Han NS, Kort R, Liu SQ, et al. Fermented foods in a global age: East meets West. Compr Rev Food Sci Food Saf. 2020;19(1):184-217.

7. Lortal S, El Mecherfi K-E, Mariotti F, Eutamène H, Rul F, Champomier-Vergès M-C, et al. Aliments fermentés \& bénéfices santé : un défi pour la recherche. Cahiers de Nutrition et de Diététique. 2020.

8. Melini F, Melini V, Luziatelli F, Ficca AG, Ruzzi M. Health-Promoting Components in Fermented Foods: An Up-to-Date Systematic Review. Nutrients. 2019;11(5).

9. Shiby VK, Mishra HN. Fermented milks and milk products as functional foods--a review. Crit Rev Food Sci Nutr. 2013;53(5):482-96.

10. Gupta RK, Gangoliya SS, Singh NK. Reduction of phytic acid and enhancement of bioavailable micronutrients in food grains. Journal of food science and technology. 2015;52(2):676-84.

11. El Mecherfi KE, Todorov SD, Cavalcanti de Albuquerque MA, Denery-Papini S, Lupi R, Haertlé T, et al. Allergenicity of Fermented Foods: Emphasis on Seeds Protein-Based Products. Foods. 2020;9(6).

12. Capozzi V, Fragasso M, Russo P. Microbiological Safety and the Management of Microbial Resources in Artisanal Foods and Beverages: The Need for a Transdisciplinary Assessment to Conciliate Actual Trends and Risks Avoidance. Microorganisms. 2020;8(2).

13. Alvarez M, Moreno-Arribas MV. The problem of biogenic amines in fermented foods and the use of potential biogenic amine-degrading microorganisms as a solution. Trends Food Sci Technol. 2014;39.

14. van Hylckama Vlieg JE, Veiga P, Zhang C, Derrien M, Zhao L. Impact of microbial transformation of food on health - from fermented foods to fermentation in the gastro-intestinal tract. Current opinion in biotechnology. 2011;22(2):211-9.

15. Ceapa C, Wopereis H, Rezaiki L, Kleerebezem M, Knol J, Oozeer R. Influence of fermented milk products, prebiotics and probiotics on microbiota composition and health. Best practice \& research Clinical gastroenterology. 2013;27(1):139-55.

16. Sanlier N, Gokcen BB, Sezgin AC. Health benefits of fermented foods. Crit Rev Food Sci Nutr. 2019;59(3):506-27.

17. Ghosh T, Beniwal A, Semwal A, Navani NK. Mechanistic Insights Into Probiotic Properties of Lactic Acid Bacteria Associated With Ethnic Fermented Dairy Products. Frontiers in microbiology. 2019;10:502.

18. Gille D, Schmid A, Walther B, Vergeres G. Fermented Food and Non-Communicable Chronic Diseases: A Review. Nutrients. 2018;10(4).

19. Peluzio M, Dias MME, Martinez JA, Milagro FI. Kefir and Intestinal Microbiota Modulation: Implications in Human Health. Frontiers in nutrition. 2021;8:638740.

20. Marx W, Scholey A, Firth J, D'Cunha NM, Lane M, Hockey M, et al. Prebiotics, probiotics, fermented foods and cognitive outcomes: A meta-analysis of randomized controlled trials. Neurosci Biobehav Rev. 2020;118:472-84. 
21. Shen J, Wilmot KA, Ghasemzadeh N, Molloy DL, Burkman G, Mekonnen G, et al. Mediterranean Dietary Patterns and Cardiovascular Health. Annu Rev Nutr. 2015;35:425-49.

22. Ndlovu T, van Jaarsveld F, Caleb OJ. French and Mediterranean-style diets: Contradictions, misconceptions and scientific facts-A review. Food research international (Ottawa, Ont). 2019;116:840-58. 23. Li N, Wu X, Zhuang W, Xia L, Chen Y, Zhao R, et al. Soy and Isoflavone Consumption and Multiple Health Outcomes: Umbrella Review of Systematic Reviews and Meta-Analyses of Observational Studies and Randomized Trials in Humans. Molecular nutrition \& food research. 2020;64(4):e1900751. 